\title{
A novel self-sensing technique for tapping-mode atomic force microscopy
}

\author{
Michael G. Ruppert ${ }^{a)}$ and S. O. Reza Moheimani ${ }^{\mathrm{b})}$ \\ The University of Newcastle, University Drive, Callaghan NSW 2308, Australia
}

(Received 16 July 2013; accepted 16 November 2013; published online 16 December 2013)

\begin{abstract}
This work proposes a novel self-sensing tapping-mode atomic force microscopy operation utilizing charge measurement. A microcantilever coated with a single piezoelectric layer is simultaneously used for actuation and deflection sensing. The cantilever can be batch fabricated with existing micro electro mechanical system processes. The setup enables the omission of the optical beam deflection technique which is commonly used to measure the cantilever oscillation amplitude. Due to the high amount of capacitive feedthrough in the measured charge signal, a feedforward control technique is employed to increase the dynamic range from less than $1 \mathrm{~dB}$ to approximately $35 \mathrm{~dB}$. Experiments show that the conditioned charge signal achieves excellent signal-to-noise ratio and can therefore be used as a feedback signal for atomic force microscopy imaging. (C) 2013 AIP Publishing LLC. [http://dx.doi.org/10.1063/1.4841855]
\end{abstract}

\section{INTRODUCTION}

In atomic force microscopy (AFM), ${ }^{1}$ high-resolution images on the nanometer scale of a wide variety of materials ${ }^{2}$ are obtained by mapping the intermolecular forces between a microcantilever with a sharp tip and a sample's surface. Tapping-mode ${ }^{3}$ AFM emerged as a popular method to minimize lateral friction forces in order to enable imaging of soft biological samples by exciting the cantilever near its first resonance frequency. While a piezoelectric tube scanner moves the sample underneath the cantilever in a raster pattern, topography features of the sample cause changes in the amplitude of the oscillating cantilever. The vibration amplitude is kept constant at a setpoint amplitude by a feedback controller through positioning the piezoelectric scanner in the vertical axis. Thus, the output of the controller can be used to estimate the sample topography and when plotted against the lateral position of the scanner, a three-dimensional image is obtained.

Silicon cantilevers have proven to be very suitable for tapping-mode AFM and additionally they can be produced economically due to the existence of a number of batch fabrication processes. ${ }^{4}$ These cantilevers are usually excited at their base by means of a dither piezoelectric transducer. However, other techniques such as photothermal, ${ }^{5,6}$ magnetic, ${ }^{7}$ or ultrasonic actuation ${ }^{8,9}$ have been demonstrated.

Despite reduced friction forces, tapping-mode AFM comes with the cost of low scan speeds due to constraints set by the bandwidth of the z-axis feedback loop and the transient response of the cantilever. Extensive research has been conducted on Q-Control ${ }^{10}$ to increase scan speed, but other methods such as scanning on higher eigenmodes ${ }^{11}$ or decreasing the size of the cantilever ${ }^{12}$ have been proposed. As most commercial AFM systems rely on displacement measurement obtained with the optical beam deflection technique, ${ }^{13,14}$ an appropriate laser position on the cantilever becomes crucial

\footnotetext{
a) Electronic mail: Michael.Ruppert@uon.edu.au

b) Electronic mail: Reza.Moheimani@ newcastle.edu.au
}

but might not be obtained at all for small cantilevers. Additional drawbacks of this method arise from possible optical interference and optical feedback ${ }^{15}$ and contribute to the measurement noise. Capacitive sensing ${ }^{16,17}$ has been introduced to reduce the size of the measurement equipment but still requires an external sensor.

Ultimately, the size and cost of the AFM system could be greatly reduced with integrated sensors or self-sensing techniques. An integrated piezoresistive element in the silicon cantilever together with a Wheatstone bridge ${ }^{18-21}$ enables near sensorless strain measurement but requires an additional coating of doped silicon. When a piezoelectric layer is deposited on one side of the cantilever, piezoelectric sensing ${ }^{22-27}$ can be used to estimate the cantilever deflection by measuring the electrical current flowing through the piezoelectric material. An implementation has been proposed using an analog circuit consisting of a voltage bridge, an instrumentation amplifier, and a bandpass filter, however hand-tuning of certain elements is necessary. ${ }^{26}$

Recently, a Frequency-Modulation AFM (FM-AFM) operation mode was reported ${ }^{27}$ using a self-sensing quartz tuning fork with an attached cantilever. ${ }^{28}$ The measured transfer function of the electrical impedance of the tuning fork, which was used for deflection sensing, showed a noticeable amount of feedthrough which was associated to a parasitic capacitance in parallel with the piezoelectric tuning fork. The feedthrough could be reduced by tuning a reference capacitor ${ }^{29}$ driven with the negated input signal of the tuning fork or by tuning an inverting gain stage in series with a reference capacitor. ${ }^{30} \mathrm{~A}$ drawback of this approach is that the piezoelectric properties of the cantilever were shown to be frequency dependent ${ }^{31}$ and thus feedthrough cancellation with static external electronics becomes difficult. Efforts have been made to address this issue by employing an external reference cantilever, ${ }^{25}$ but even two cantilevers from the same batch will have slightly varying piezoelectric capacitances. ${ }^{25}$

Alternative methods based on magnetoresistive sensing ${ }^{32}$ and tunnel magnetoresistive sensing ${ }^{33}$ promise high bandwidth and resolution but require additional actuators for 


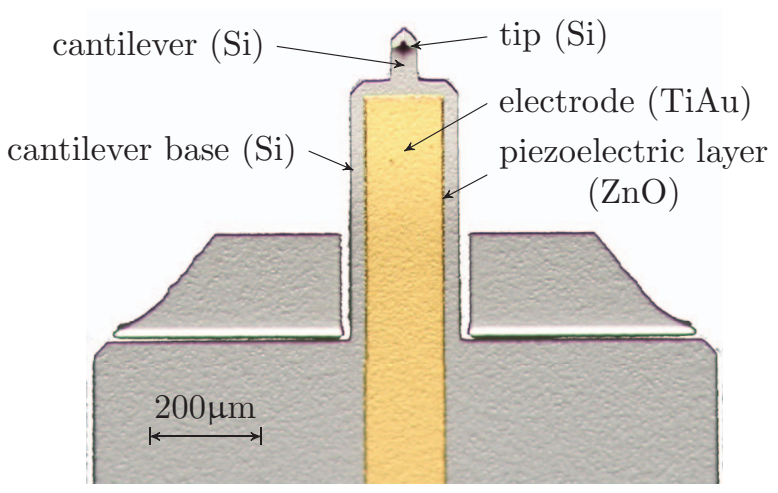

FIG. 1. Image of piezoelectric microcantilever.

cantilever excitation as well as the generation of a homogeneous magnetic field complicating the design. Cantilevers with thermal heating loops located at the tip of the cantilever for actuation and a piezoresistive element at the base of the cantilever for sensing ${ }^{34,35}$ have also been proposed but major control effort to compensate for coupling has to be employed.

This contribution proposes self-sensing tapping-mode AFM with a cantilever shown in Fig. 1 that utilizes a single piezoelectric $(\mathrm{ZnO})$ layer for actuation and sensing. The silicon cantilever can be cost-effectively fabricated with existing MEMS processes. A charge measurement circuit in combination with a signal conditioning block is used to effectively extract an estimate of the cantilever's deflection without the optical laser sensor. Capacitive feedthrough cancellation is systematically obtained over a band of frequencies around the resonance with the use of a feedforward controller.

The remainder of the paper is organized as follows. In Sec. II, the equivalent electromechanical actuator/sensor model of the cantilever is discussed. The resulting transfer function from applied actuator voltage to measured charge contains a significant amount of feedthrough, decreasing the dynamic range of the system. In Sec. III, the circuit to measure charge while the cantilever is being actuated by an AC voltage is discussed. A method is proposed in Sec. IV to calculate a feedforward controller to cancel the feedthrough arising from the piezoelectric capacitance. In Sec. V, the conditioned charge signal is compared with the laser signal in terms of signal-to-noise ratio and scan experiments are shown which validate charge can be used effectively as a feedback signal for topography estimation. To conclude, Secs. VI and VII summarize the results and outline the possible future trend towards on-chip AFM imaging.

\section{ACTUATOR/SENSOR MODEL OF CANTILEVER}

The system under consideration is a cantilevered beam with a piezoelectric layer bonded to its surface acting as a self-sensing actuator as shown in Fig. 2(a). Assuming perfect bonding between the piezoelectric patch and the beam, a voltage applied to the terminals of the piezoelectric layer results in a bending moment causing the cantilever to deflect. The transfer function relating the piezoelectric actuator voltage $V(s)$ to cantilever deflection $D(s)$, only considering the

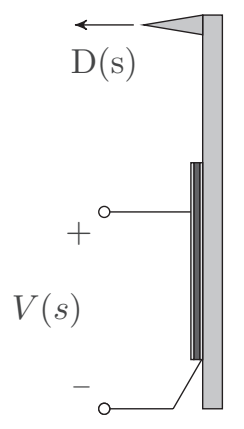

(a)

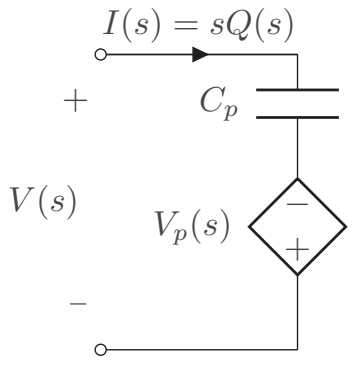

(b)
FIG. 2. (a) Simplified schematic and (b) equivalent electrical circuit model of the piezoelectric cantilever.

fundamental mode, is given by Ref. 36

$$
G_{d v}(s)=\frac{D(s)}{V(s)}=\frac{\alpha \omega_{0}^{2}}{s^{2}+\frac{\omega_{0}}{Q} s+\omega_{0}^{2}} .
$$

Similarly, when a poled piezoelectric transducer is subjected to mechanical strain it becomes electrically polarized, producing a charge on the surface of the material. This direct piezoelectric effect can be modeled as a strain dependent voltage source $V_{p}$ in series with a capacitor $C_{p}$ as shown in Fig. 2(b). While the capacitor sufficiently represents the dielectric properties of the piezoelectric material, this simplified model does not take into account dielectric losses or heat dissipation which can be modeled by adding a resistor in parallel to $V_{p}$ and $C_{p}$. The model is a simplified version of the Butterworth Van Dyke model as proposed by the IEEE Standard on Piezoelectricity. ${ }^{37}$ Since the voltage $V_{p}$ is dependent on the strain in the structure, the transfer function from the voltage applied to the terminals of the piezoelectric transducer to the voltage induced in the sensor $V_{p}$ is given by Ref. 36

$$
G_{v v}(s)=\frac{V_{p}(s)}{V(s)}=\frac{\beta \omega_{0}^{2}}{s^{2}+\frac{\omega_{0}}{Q} s+\omega_{0}^{2}} .
$$

Applying Kirchhoff's law to Fig. 2(b), one obtains

$$
V(s)=\frac{1}{C_{p}} Q(s)-V_{p}(s) .
$$

Substituting (2) into (3) yields

$$
G_{q v}(s)=\frac{Q(s)}{V(s)}=C_{p}+C_{p} G_{v v}(s) .
$$

Observing that (1) and (2) only differ by a constant factor, (4) can be rewritten as

$$
G_{q v}(s)=C_{p}+C_{p} \gamma G_{d v}(s) .
$$

From (5), we may conclude that by measuring charge at the terminals of the piezoelectric layer, an estimate of the cantilever deflection can be derived.

\section{CHARGE MEASUREMENT}

The circuit shown in Fig. 3 has been designed to measure the charge in the piezoelectric transducer while applying a voltage signal at resonance. The charge mode amplifier will equate the charge present at its negative input by charging 


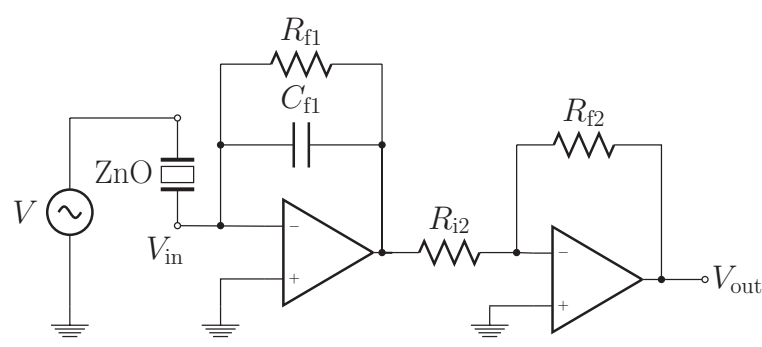

FIG. 3. Charge measurement circuit.

the feedback capacitor $C_{\mathrm{f} 1}$. A feedback resistor $R_{\mathrm{f} 1}$ is used to prevent the amplifier from drifting into saturation. In order to obtain a higher gain and to accommodate for the inverting character of the charge mode amplifier, an inverting amplifier is added in series. The transfer function of the circuit is given by

$$
H(s)=\frac{V_{\text {out }}(s)}{V_{\text {in }}(s)}=\frac{a s}{s+\omega_{c}},
$$

resembling a high-pass filter with a cut-off frequency of $\omega_{c}=\frac{1}{R_{\mathrm{f} 1} C_{\mathrm{f} 1}}$ and a gain of $a=\frac{R_{\mathrm{f} 2}}{R_{\mathrm{i} 2} C_{\mathrm{f} 1}}$. The values are chosen such that the frequency of interest (the resonance frequency of the first eigenmode of the cantilever) lies well within the bandwidth of the charge sensor and such that the amplitude of the charge signal is of the same order of magnitude as the corresponding laser signal. The circuit was implemented on a prototype board with standard components and LT1468 operational amplifiers and was used to obtain $G_{q v}$ shown in Fig. 4. It is obvious that $G_{q v}$ is heavily buried in feedthrough, thus decreasing the dynamic range to less than $1 \mathrm{~dB}$ compared to approximately $30 \mathrm{~dB}$ range of $G_{d v}$. This is of great disadvantage when using the charge signal as an amplitude estimate for tapping-mode AFM since a shift in the resonance frequency caused by interaction forces will yield a very small change in oscillation amplitude resulting in poor resolution.

\section{FEEDTHROUGH CANCELLATION}

MEMS devices are known to be prone to parasitic feedthrough due to capacitive stray circuits in the system. This is usually the case because drive and sense electrodes share a

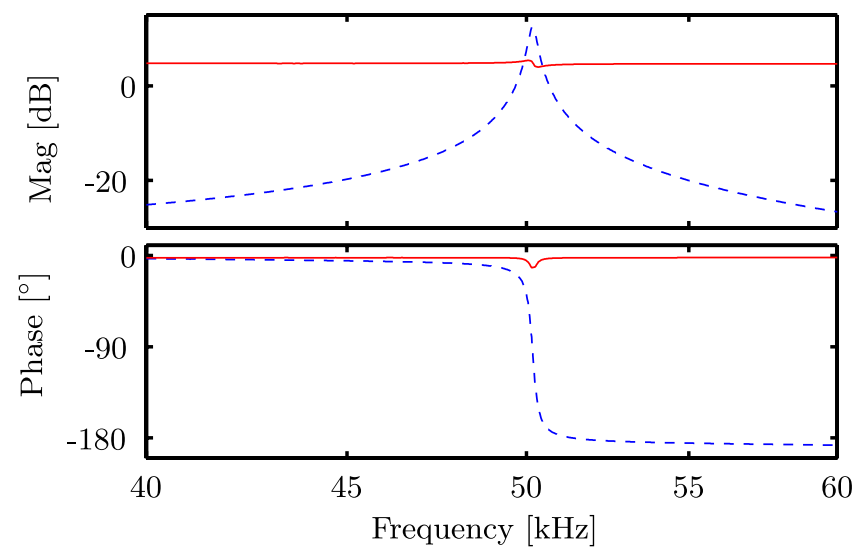

FIG. 4. Measured FRF of $G_{d v}(--)$ and $G_{q v}(-)$. common node (the MEMS electrical network). ${ }^{38}$ When capacitive sensing is used for position sensing of the MEMS device, the parasitic feedthrough current has to be separated from the motional current. A number of actuation and cancellation techniques ${ }^{39}$ have been proposed that rely on differential driving of an external tunable capacitor, ${ }^{40}$ second harmonic actuation, ${ }^{41}$ and piezoresistive sensing. ${ }^{42}$ In the electromechanical amplitude modulation (EAM) technique, the feedthrough current can be separated from the motional signal in the frequency domain by modulating the common node of the MEMS device with a high frequency carrier signal. ${ }^{43}$ An additional external capacitor that is driven out of phase to the modulating signal can be used to further minimize the feedthrough current. ${ }^{44}$

As can be seen from (5) and Fig. 4, the dynamics of the cantilever are heavily buried in capacitive feedthrough resulting in a very low dynamic range around resonance. When the charge signal is used as a feedback signal for AFM imaging, it has to be conditioned such that the dynamic range is increased. For this purpose, a feedforward controller $K(s)$ is derived and implemented on a Field Programmable Analog Array (FPAA). The Anadigm AN221E04 FPAA offers configurable filter and summing blocks with a bandwidth of up to $400 \mathrm{kHz}$ and are therefore well suited for this application. However, due to the interfacing circuitry and the switched capacitor nature of the FPAA board, additional signal attenuation and phase shifts have to be accommodated for in the design of $K(s)$. Fig. 5 shows the schematic setup of the charge measurement and feedthrough cancellation where $H(s)$ resembles the charge measurement circuit and $F_{1}(s)$ and $F_{2}(s)$ have been introduced to model the signal attenuation through the FPAA board. The controller $K(s)$ can be calculated as

$$
K(s)=\frac{C_{p} H(s) F_{1}(s)}{F_{2}(s)} .
$$

With $H(s), F_{1}(s)$, and $F_{2}(s)$ all being first order transfer functions, the resulting feedforward controller will be of order three. To simplify the design, a linear transfer function is fitted to $K(s)$ in a frequency range around the resonance. The closed loop simulation shown in Fig. 6 verifies the feedthrough cancellation with the full-order $K(s)$ and linear approximation around the resonance.

\section{EXPERIMENTAL RESULTS}

\section{A. System identification}

The AFM cantilever used in this work is the Dimension Micro-Actuated Silicon Probe (DMASP), a piezoelectric

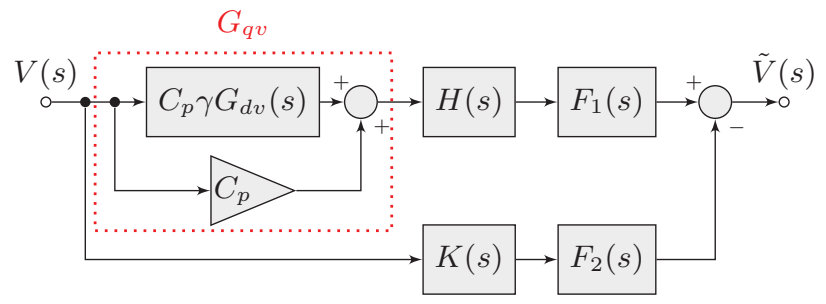

FIG. 5. Block diagram showing the schematic setup of charge measurement and feedthrough cancellation. 


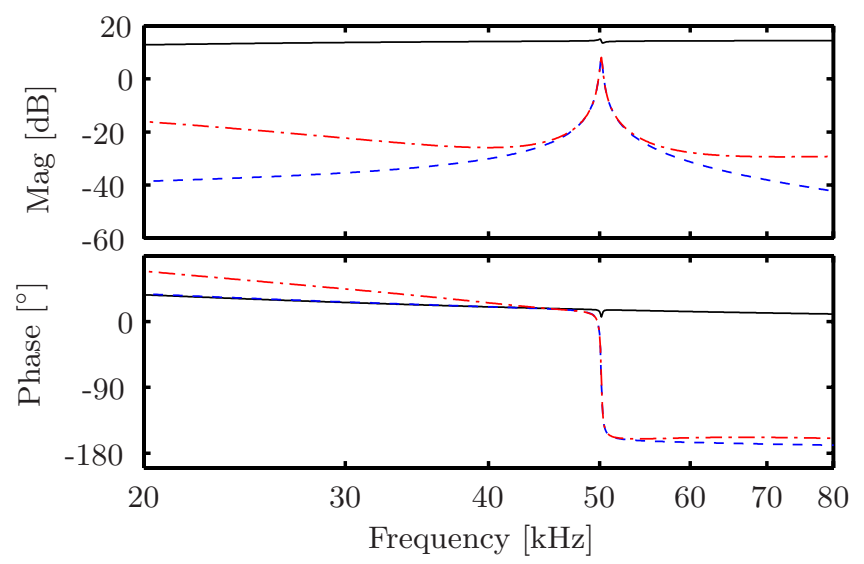

FIG. 6. Simulation of open loop (-), closed loop with $K(s)(--)$, and with linear approximation of $K(s)(-\cdot-)$.

self-actuated microcantilever manufactured by Bruker. A model of $G_{d v}$ was directly obtained using the frequency domain subspace identification algorithm. ${ }^{45}$ The parameters of $G_{q v}$ are found by appropriate scaling and nonlinear optimization over $C_{p}$ and $\gamma$ to minimize the difference in magnitude and phase of the measured transfer function of $G_{q v}$ and (5). The resulting parameters are shown in Table I. Additionally, knowing that the parameters are inherent properties of the piezoelectric cantilever, a precision LCR meter such as the Agilent E4980A was used to measure $C_{p}$ and the obtained value of $23.78 \mathrm{pF}$ adequately matches the estimation.

\section{B. Feedthrough cancellation}

With the above design procedure, tuning of the controller parameters is reduced to merely adjusting the gain of the implemented transfer function of the FPAA. Fig. 7 demonstrates how adjusting the gain leads to increased cancellation of the feedthrough term. It can be seen that the dynamic range is increased and the zero is pushed to higher frequencies when good matching is achieved. When the gain of $K(s)$ is too high the zero appears on the other side of the resonance. As can be seen from Fig. 7 the dynamic range is increased to around $35 \mathrm{~dB}$ and the phase crosses $-90^{\circ}$ at resonance and almost reaches $-180^{\circ}$ after the resonance.

\section{Noise comparison}

A noise comparison of the laser and charge signal demodulated at the resonance frequency is conducted with a lock-in amplifier and shown in Fig. 8. The microcantilever is excited at its resonance frequency with $200 \mathrm{mV}(\mathrm{p})$ and the signal-tonoise ratio (SNR) is measured around a bandwidth of $100 \mathrm{~Hz}$. The SNR is determined to be the difference between the maximum value and the fitted noise floor. For the laser signal, the

TABLE I. Parameter of identified model.

\begin{tabular}{cccccc}
\hline \hline Mode shape & $f_{0}[\mathrm{kHz}]$ & $Q$ & $\beta[\mu \mathrm{m} / \mathrm{V}]$ & $C_{p}[\mathrm{pF}]$ & $\gamma[\mathrm{V} / \mu \mathrm{m}]$ \\
\hline & 50.2 & 219.3 & 0.02 & 22.3 & 0.04 \\
\hline
\end{tabular}

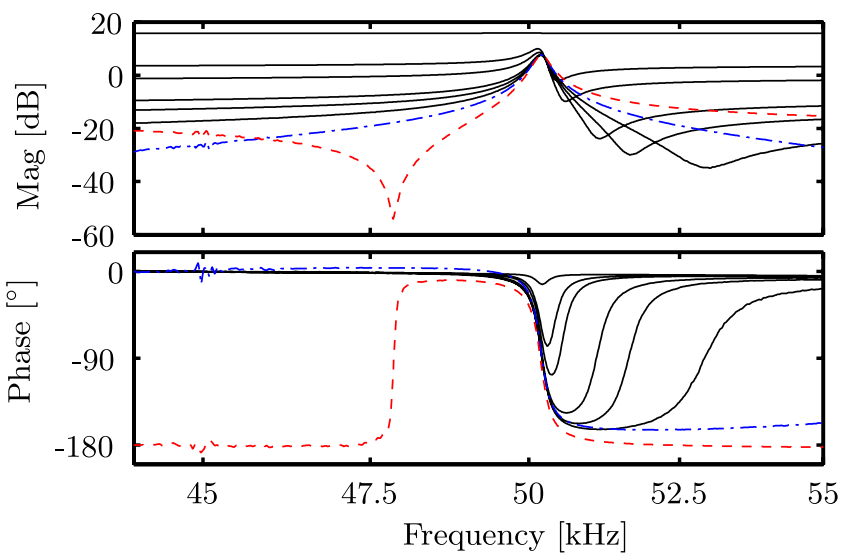

FIG. 7. Measured $G_{q v}$ with undercompensated (-), compensated $(-\cdot-)$, and overcompensated (--) feedthrough.

SNR is determined to be approximately $134 \mathrm{~dB}$ and for the charge signal approximately $116 \mathrm{~dB}$. The specification of the measurement instrument states an input amplitude noise of approximately $-166 V_{\mathrm{dB}} / \sqrt{\mathrm{Hz}}{ }^{46}$ The noise floor of the demodulated signal is around $18 \mathrm{~dB}$ higher than that of the laser signal. This can be attributed to imperfections of the charge measurement circuit, which was implemented on a prototype stripboard. Also, it was found that the circuit, being of very high gain by design, is very sensitive to interferences emanating from ambient sources such as from the electrical ballasts in fluorescent lamps and therefore needed a protective shield. This specific noise source can be traced in Fig. 7 in the form of frequency components around $45 \mathrm{kHz}$ when the shielding is insufficient. In future work, the authors expect to further improve the SNR of the demodulated charge signal by using a printed circuit board (PCB) design with surface mount components and improved shielding.

In Fig. 9, the time domain signal of the laser and charge sensor when the cantilever is oscillating in free air are compared. It can be seen that the charge signal shows a superimposed oscillation which can be associated to the $45 \mathrm{kHz}$ disturbance originating from ambient lighting. As mentioned before, this noise source can be minimized by electrostatically shielding the circuitry. When analyzing the amplitude spectra

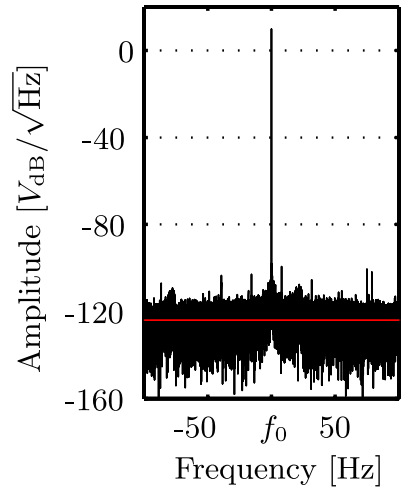

(a)

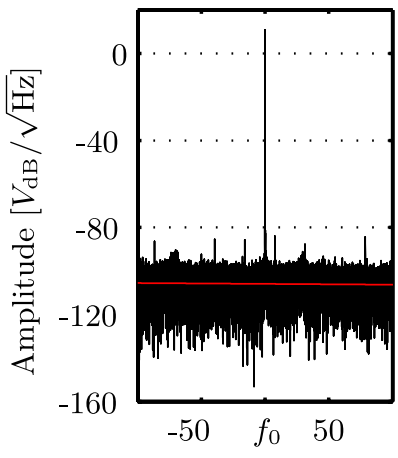

Frequency $[\mathrm{Hz}]$

(b)
FIG. 8. Zoom-FFT of (a) the laser signal and (b) the charge signal normalized around the resonance frequency. The horizontal red line indicates the fitted noise floor. 

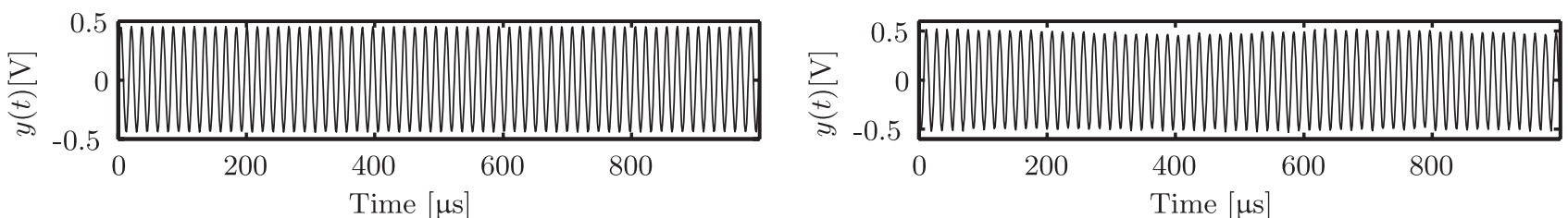

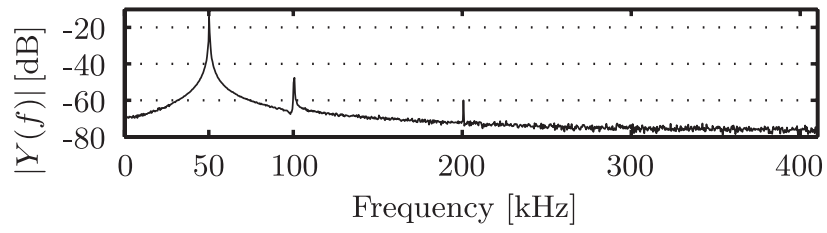

(a)

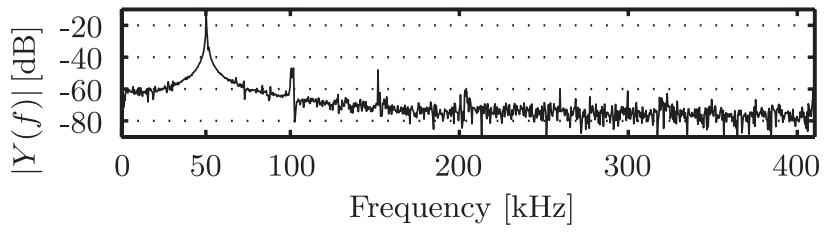

(b)

FIG. 9. Time-domain and single-sided amplitude spectrum of (a) laser signal and (b) charge signal while oscillating at the resonance frequency in free air.

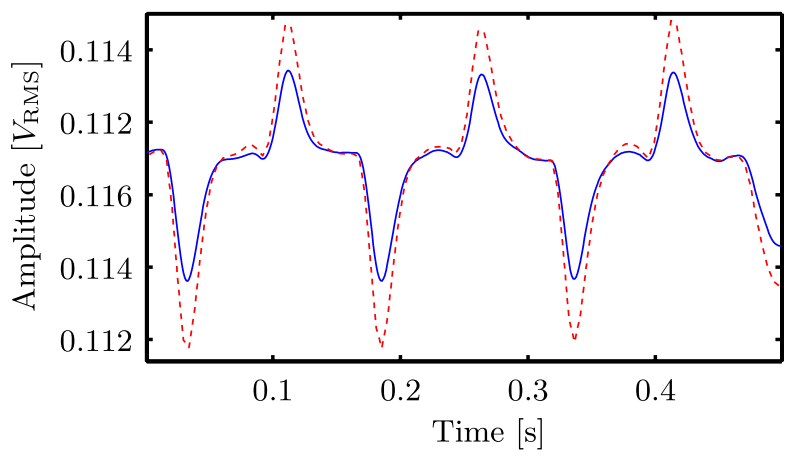

FIG. 10. Demodulated laser signal (-) and demodulated charge signal (--) corresponding to the error signal of the $\mathrm{z}$-axis controller during scanning.
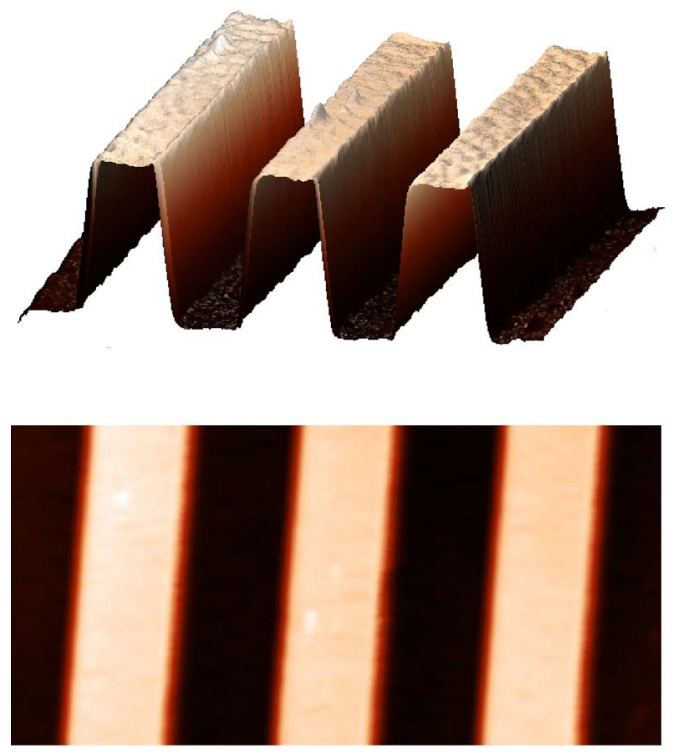

(a) of the two signals, the charge signal shows a slightly higher noise floor but a similarly strong component at the first resonance compared to the laser signal.

While tapping on a sample additional noise may be induced via capacitive coupling between the $\mathrm{ZnO}$ layer and the sample. For the cantilever used in this work, the $\mathrm{ZnO}$ patch approximately has a thickness, length, and width of $1 \mu \mathrm{m}$, $1200 \mu \mathrm{m}$, and $170 \mu \mathrm{m}$, respectively. Assuming a dielectric constant of $\epsilon_{r}=11$ for the $\mathrm{ZnO}$ film, the capacitance between the electrodes can be calculated as $C_{p} \approx 19.9 \mathrm{pF}$, which is close to the measured and identified value from Table I. Assuming that in the worst case scenario the cantilever is parallel above the sample and only separated by the tip length $(15-20 \mu \mathrm{m})$ and free air, the capacitance between the sample and the $\mathrm{ZnO}$ layer is more than two orders of magnitude smaller than $C_{p}$ and can therefore be neglected.

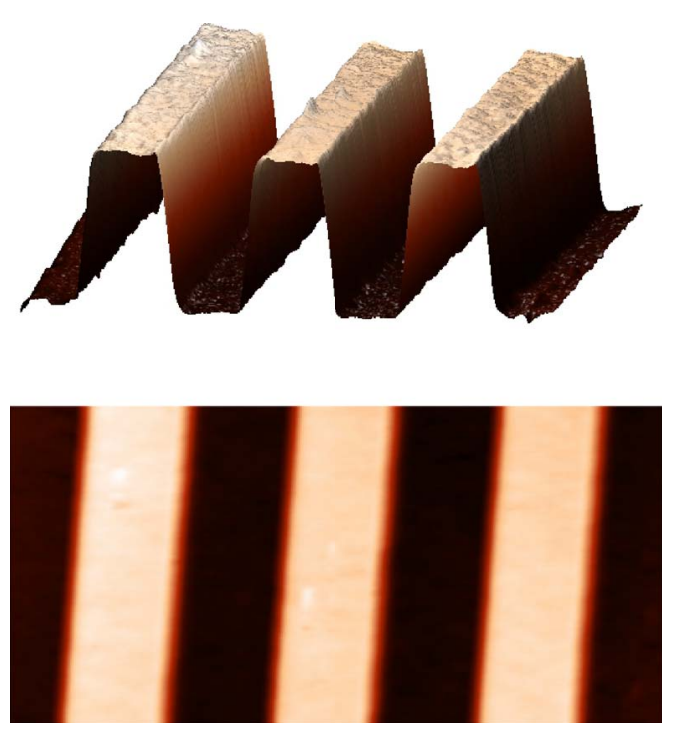

(b)

FIG. 11. 3D and 2D images of scan of the TGZ1 calibration grating with (a) the laser sensor and (b) the charge sensor used as the feedback signal for topography estimation. 


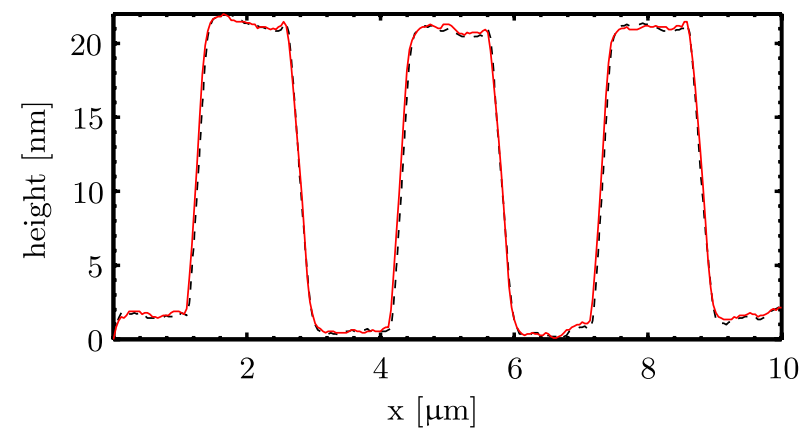

FIG. 12. Cross-section analysis of image obtained with the laser sensor $(--)$ and the charge sensor $(-)$.

\section{Scan results}

Scan experiments with a NT-MDT NTEGRA Prima AFM on a NT-MDT TGZ1 calibration grating have been conducted. The sample contains periodic features with step heights of $21.6 \pm 1.5 \mathrm{~nm}$. While scanning the calibration grating the demodulated amplitude signal of the laser and charge sensor are compared. When the z-axis feedback loop is closed, the demodulated amplitude signal corresponds to the error signal which is kept at the setpoint amplitude by the z-axis controller. As can be seen from Fig. 10 charge and laser signals correspond very well, the only difference being the magnitude of the error peaks which is due to the gain in the charge measurement circuitry. Since the operating amplitude setpoint of the scanning procedure is set relative to the free air amplitude, this is of no concern. Fig. 11 shows images obtained with the laser signal and charge signal as the deflection measurement. Both scans were conducted over the same area of $10 \mu \mathrm{m} \times 10 \mu \mathrm{m}$ at a speed of $20 \mu \mathrm{m} / \mathrm{s}$. The setpoint amplitude is kept at $50 \%$ of the free-air amplitude and the zaxis feedback gain is kept constant for both scans as well. As can be seen there is literally no difference in image quality when using the charge signal instead of the laser signal. Additionally, a cross-section analysis of the 3D images obtained is shown in Fig. 12 which highlights the equal image quality obtained with the charge signal.

\section{CONCLUSIONS}

This work has highlighted how the optical deflection measurement system used in tapping-mode atomic force microscopy can be easily replaced with an alternative sensor mechanism when a cantilever equipped with a piezoelectric transducer is used. A single piezoelectric layer can be used simultaneously as a voltage-driven actuator and as a charge-measuring sensor. The readout circuit is simple and can be realized with only two operational amplifiers. The capacitive feedthrough, which is noticeable in the voltage to charge transfer function, and significantly degrades the dynamic range, is systematically canceled by using a feedforward controller. A FPAA based implementation is proposed that offers flexibility and sufficient bandwidth over the desired frequency range. It was shown that the charge signal achieves an excellent signal-to-noise ratio, only a few $\mathrm{dB}$ less than the laser signal. When charge is used as a feedback signal for topography estimation during a tapping-mode AFM experiment, the obtained images are of identical quality compared to imaging with the laser signal.

\section{FUTURE WORK}

In future work, the authors aim to further increase the signal-to-noise ratio of the charge signal by improving the analog circuitry. Moreover, it would be of great interest to devise an automatic feedthrough cancellation system using estimator design methods. With the self-sensing approach highlighted in this work, the idea of on-chip AFM seems more and more feasible. An electrostatically actuated two degree of freedom (2-DOF) nanopositioning stage based on MEMS technology is already available ${ }^{47}$ which offers low power consumption combined with a minimized footprint, but can still scan over a large range. An active on-chip cantilever integrated with a 2-DOF positioning stage has also been proposed. ${ }^{48}$ When combined with sensorless Q-Control methods, ${ }^{49,50}$ the proposed self-sensing technique can lead to a stand alone on-chip AFM.

${ }^{1}$ G. Binnig, C. F. Quate, and C. Gerber, Phys. Rev. Lett. 56, 930 (1986).

${ }^{2}$ D. Sarid, in Scanning Force Microscopy with Applications to Electric, Magnetic and Atomic Forces, edited by D. Sarid (Oxford University Press, 1994).

${ }^{3}$ Q. Zhong, D. Inniss, K. Kjoller, and V. Elings, Surf. Sci. 290, L688 (1993).

${ }^{4}$ T. Albrecht, S. Akamine, T. E. Carver, and C. Quate, J.Vac. Sci. Technol. A 8, 3386 (1990).

${ }^{5}$ H. Yamashita, N. Kodera, A. Miyagi, T. Uchihashi, D. Yamamoto, and T. Ando, Rev. Sci. Instrum. 78, 083702 (2007).

${ }^{6}$ S. W. Stahl, E. M. Puchner, and H. E. Gaub, Rev. Sci. Instrum. 80, 073702 (2009).

${ }^{7}$ W. Han, S. M. Lindsay, and T. Jing, Appl. Phys. Lett. 69, 4111 (1996).

${ }^{8}$ K. Yamanaka and S. Nakano, Jpn. J. Appl. Phys. 35, 3787 (1996).

${ }^{9}$ A. Vinckier, F. Hennau, K. Kjoller, and L. Hellemans, Rev. Sci. Instrum. 67, 387 (1996).

${ }^{10}$ J. Mertz, O. Marti, and J. Mlynek, Appl. Phys. Lett. 62, 2344 (1993).

${ }^{11}$ M. G. Ruppert, M. W. Fairbairn, and S. O. R. Moheimani, in Proceedings of the IEEE/ASME International Conference on Advanced Intelligent Mechatronics, Wollongong, Australia, 2013.

${ }^{12}$ D. A. Walters, J. P. Cleveland, N. H. Thomson, P. K. Hansma, M. A. Wendman, G. Gurley, and V. Elings, Rev. Sci. Instrum. 67, 3583 (1996).

${ }^{13}$ G. Meyer and N. M. Amer, Appl. Phys. Lett. 53, 1045 (1988).

${ }^{14}$ S. Alexander, L. Hellemans, O. Marti, J. Schneir, V. Elings, P. K. Hansma, M. Longmire, and J. Gurley, J. Appl. Phys. 65, 164 (1989).

${ }^{15}$ R. Kassies, K. O. van der Werf, M. L. Bennink, and C. Otto, Rev. Sci. Instrum. 75, 689 (2004)

${ }^{16}$ T. Goddenhenrich, H. Lemke, U. Hartmann, and C. Heiden, J. Vac. Sci. Technol. A 8, 383 (1990).

${ }^{17}$ N. Blanc, J. Brugger, N. F. de Rooij, and U. Durig, J. Vac. Sci. Technol. B 14, 901 (1996).

${ }^{18}$ M. Tortonese, R. C. Barrett, and C. F. Quate, Appl. Phys. Lett. 62, 834 (1993).

${ }^{19}$ R. Linnemann, T. Gotszalk, I. W. Rangelow, P. Dumania, and E. Oesterschulze, J. Vac. Sci. Technol. B 14, 856 (1996).

${ }^{20}$ T. Gotszalk, P. Grabiec, and I. W. Rangelow, Ultramicroscopy 82, 39 (2000).

${ }^{21}$ H. Bhaskaran, M. Li, D. Garcia-Sanchez, P. Zhao, I. Takeuchi, and H. X. Tang, Appl. Phys. Lett. 98, 013502 (2011).

${ }^{22} \mathrm{~J}$. Tansock and C. Williams, Ultramicroscopy 42-44(part 2), 1464 (1992).

${ }^{23}$ T. Itoh and T. Suga, Nanotechnology 4, 218 (1993).

${ }^{24}$ P.-F. Indermühle, G. Schürmann, G.-A. Racine, and N. F. de Rooij, Sens. Actuators, A 60, 186 (1997).

${ }^{25}$ C. Lee, T. Itoh, and T. Suga, Sens. Actuators, A 72, 179 (1999).

${ }^{26}$ J. Adams, L. Manning, B. Rogers, M. Jones, and S. Minne, Sens. Actuators, A 121, 262 (2005). 
${ }^{27}$ D. Trumper, R. Hocken, D. Amin-Shahidi, D. Ljubicic, and J. Overcash, in Control Technologies for Emerging Micro and Nanoscale Systems, Lecture Notes in Control and Information Sciences Vol. 413, edited by E. Eleftheriou and S. Moheimani (Springer, Berlin, 2011), pp. 17-46.

${ }^{28}$ T. Akiyama, U. Staufer, N. F. de Rooij, P. Frederix, and A. Engel, Rev. Sci. Instrum. 74, 112 (2003).

${ }^{29}$ Nanosensors, "Akiyama-probe technical guide," Version 2009-03-23 (Nanosensors, 2009)

${ }^{30}$ T. Akiyama, N. F. de Rooij, U. Staufer, M. Detterbeck, D. Braendlin, S. Waldmeier, and M. Scheidiger, Rev. Sci. Instrum. 81, 063706 (2010).

${ }^{31}$ J.-F. Li, D. Viehland, C. Lakeman, and D. Payne, J. Mater. Res. 10, 1435 (1995).

${ }^{32}$ D. R. Sahoo, A. Sebastian, W. Hberle, H. Pozidis, and E. Eleftheriou, Nanotechnology 22, 145501 (2011).

${ }^{33}$ A. Tavassolizadeh, T. Meier, K. Rott, G. Reiss, E. Quandt, H. Hölscher, and D. Meyners, Appl. Phys. Lett. 102, 153104 (2013).

${ }^{34}$ G. E. Fantner, D. J. Burns, A. M. Belcher, I. W. Rangelow, and K. YoucefToumi, ASME J. Dyn. Syst., Meas., Control 131, 061104 (2009)..

${ }^{35}$ G. E. Fantner, W. Schumann, R. J. Barbero, A. Deutschinger, V. Todorov, D. S. Gray, A. M. Belcher, I. W. Rangelow, and K. Youcef-Toumi, Nanotechnology 20, 434003 (2009).

${ }^{36}$ S. O. R. Moheimani and A. J. Fleming, Piezoelectric Transducers for Vibration Control and Damping (Springer-Verlag London Limited, 2006).

${ }^{37}$ IEEE Standard on Piezoelectricity, ANSI/IEEE Standard 176-1987 (1987).

${ }^{38}$ J. Clark, W.-T. Hsu, M. Abdelmoneum, and C.-C. Nguyen, J. Microelectromech. Syst. 14, 1298 (2005).
${ }^{39}$ A.-H. Lin, J.-Y. Lee, J. Yan, and A. Seshia, Sens. Actuators, A 158, 263 (2010).

${ }^{40}$ J.-Y. Lee and A. Seshia, Sens. Actuators, A 156, 36 (2009).

${ }^{41}$ V. Logeeswaran, F. Tay, M. Chan, F. Chau, and Y. Liang, Analog Integr. Circuits Signal Process. 37, 17 (2003).

42 J. van Beek, P. Steeneken, and B. Giesbers, in Proceedings of the IEEE International Frequency Control Symposium and Exposition, 4-6 June 2006, pp. $475-480$

${ }^{43}$ J. Cao and C. T.-C. Nguyen, in Digest of Technical Papers, Proceedings of the 10th International Conference on Solid-State Sensors and Actuators, Sendai, Japan, 7-10 June 1999, pp. 1826-1829.

${ }^{44}$ B. Cagdaser, A. Jog, M. Last, B. S. Leibowitz, L. Zhou, E. Shelton, K. S. Pister, and B. E. Boser, in Proceedings of the Solid-State Sensor, Actuator and Microsystems Workshop, Hilton Head Island, SC, USA, 6 June 2004 (CiteSeer, 2004), Vol. 10.

${ }^{45}$ T. McKelvey, in Proceedings of the American Control Conference, 30 June-2 July 2004, Vol. 1, pp. 673-678.

${ }^{46}$ Zürich Instruments, "HF2LI lock-in amplifier," HF2 User Manual, Revision 15296 (Zürich Instruments, 2012).

${ }^{47}$ A. G. Fowler, A. Laskovski, A. C. Hammond, and S. O. R. Moheimani, J. Microelectromech. Syst. 21, 771 (2012).

${ }^{48}$ J. Dong and P. Ferreira, J. Microelectromech. Syst. 18, 641 (2009).

${ }^{49}$ M. Fairbairn, S. Moheimani, and A. J. Fleming, J. Microelectromech. Syst. 20, 1372 (2011).

${ }^{50}$ M. W. Fairbairn and S. O. R. Moheimani, Rev. Sci. Instrum. 84, 053706 (2013). 